\title{
A Prediction Method of Charging Station Planning Based on BP Neural Network
}

\author{
Jia Xu, Jing Li, Xin Liao, Changping Song \\ School of Computer Science and Technology, Shanghai University of Electric Power, Shanghai, China \\ Email: 1540651819@qq.com 2 lijing@shiep.edu.cn
}

How to cite this paper: $\mathrm{Xu}, \mathrm{J} ., \mathrm{Li}, \mathrm{J} ., \mathrm{Liao}$ X. and Song, C.P. (2019) A Prediction Method of Charging Station Planning Based on BP Neural Network. Journal of Computer and Communications, 7, 219-230. https://doi.org/10.4236/jcc.2019.77018

Received: June 10, 2019

Accepted: July 21, 2019

Published: July 24, 2019

Copyright (อ 2019 by author(s) and Scientific Research Publishing Inc. This work is licensed under the Creative Commons Attribution International License (CC BY 4.0).

http://creativecommons.org/licenses/by/4.0/

(c) (i) Open Access

\begin{abstract}
The construction of charging service facilities is a very important factor in the popularization of electric vehicles. Therefore, the planning problems of electric vehicle charging station are urgent to be solved. Considering the standard of natural environment, society, traffic, power grid and economy, an evaluation system is created for electric vehicle charging station project through 15 sub-standards. Planning model of charging station is constructed based on BP neural network adopted in the analysis. It is used for location and capacity prediction of charging station planning. By analyzing the model with data samples, a stable network structure is established and the feasibility of the model is verified in the charging station planning.
\end{abstract}

\section{Keywords}

Electric Vehicle Charging Station, BP Neural Network, Location, Capacity Prediction

\section{Introduction}

Emissions from fuel vehicles are one of the most important causes of environmental pollution. In order to reduce environmental pollution, governments advocate green travel like carrying on impose restriction on car license number in many countries, which has brought a lot of inconvenience to the public. If pollution caused by fuel vehicles is required to be solved fundamentally, we must replace the fuel vehicles with new energy vehicles. Some Western countries who signed the "Paris Agreement" have set a timetable for fuel vehicle sales ban: Norway's deadline is 2025, India is 2030 and Britain and France are 2040. The Paris plans to ban the use of diesel locomotives including diesel and petrol by 2030. The Deputy Minister of the Ministry of Industry and Information Technology of China also pointed out that China will consider setting a timetable for 
the ban on fuel vehicles, and it is imperative to develop new energy vehicles.

New energy vehicles can effectively reduce the deterioration of environmental pollution and adopting new energy vehicles is an effective way to solve the depletion of fossil resources. Electric vehicles play an important role. With the continuous improvement of battery technology, electric vehicles have become the center of the development trend of new energy vehicles. Since 2009, China's subsidy policy for new energy vehicles has been going on for ten years. As shown in Figure 1, the sales of new energy vehicles are showing a rapid growth trend due to the government subsidy policy. The popularity of new energy vehicles is unquestionable with the largest share of electric vehicles and pure electric vehicles accounting for at least $80 \%$ of new energy vehicles. However, basic charging service facilities of electric vehicles in China are still the shortcomings of the development of new energy vehicles. The construction of charging service facilities is a very important factor in the popularization of electric vehicles. Therefore, the planning problems of electric vehicle charging station are urgent to be solved.

The location and capacity prediction are most part in the planning and its problems are generally regarded as an optimization problem. Literature [1] provides a new research perspective by considering uncertainty, qualitative and quantitative factors into the site selection assessment, and presents the mainstream penetration of $\mathrm{BN}$ as a powerful decision-making tool in the context of electrical energy management. According to the actual measured vehicle arrival heat map data, the literature [2] establishes a charging load estimation model and introduces a multi-objective planning model, which takes into account the actual operational constraints of the distribution network. The literature [3] mainly studies the location division of urban charging stations, determines the power supply range, and combines the traditional Voronoi diagram and real-time traffic flow map information. Literature [4] uses POI-specific geographic influences to

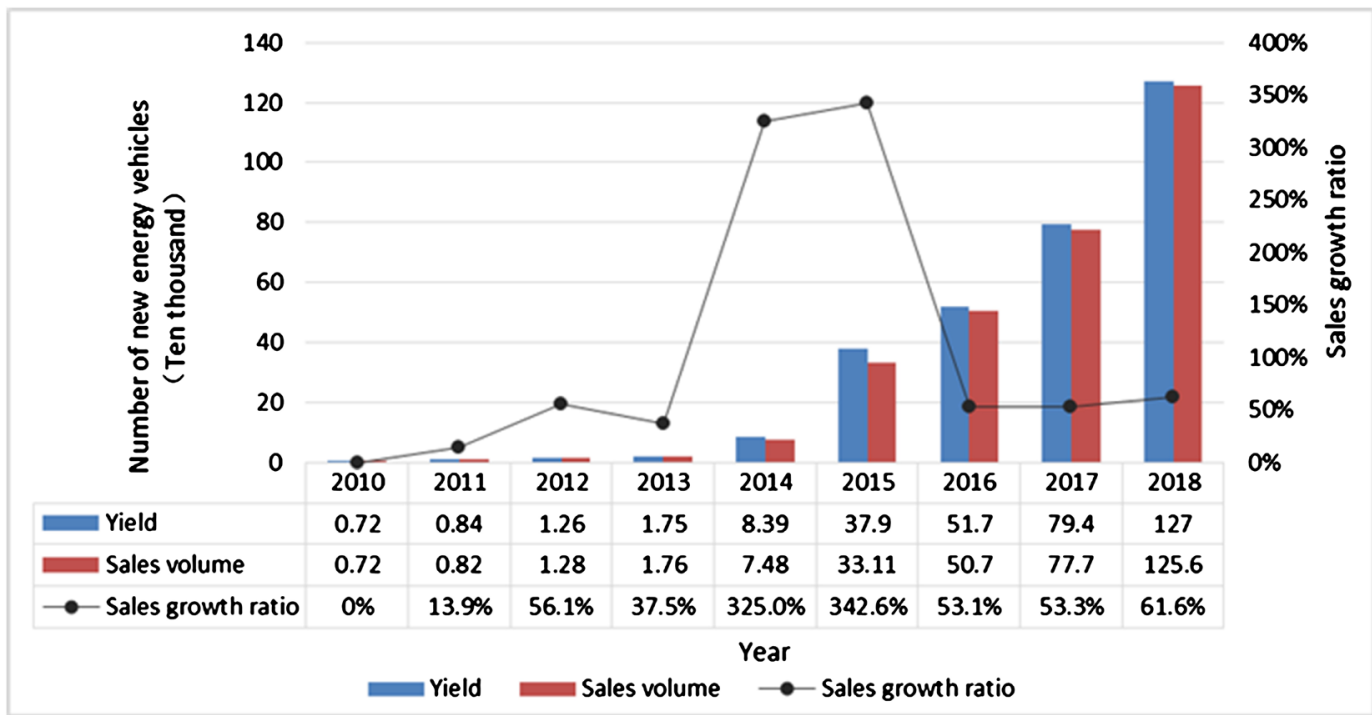

Figure 1. The growth trend of new energy vehicles. 
improve POI recommendations. We use three factors to simulate the geographic impact between two POIs: the geographic impact of POI, the geographic sensitivity of POIs, and their physical distance. Literature [5] establish a path selection model and an assessment model of urban traffic satisfaction. Considering the fact that DGs have to supply power to distribution network and EV charging stations simultaneously, a model to determine the site and capacity of DGs and EV charging stations, in which the minimum total cost, the lowest network loss and the highest traffic satisfaction are taken as the objectives, is built and solved by new multi-objective free-search algorithm, and then a Pareto solution set is achieved. Literature [6] presents a location method of electric vehicle charge station based on data driven. Potential traffic demand, including traffic origin and destination are extracted with massive location data. Map is divided into grids with the same interval. Traffic demand of each grid is calculated. Grids with large traffic demand are selected as the alternative location for electric charging station.

Previous researches have focused on economic and social standards such as financial constraints and service capacity constraints, construction costs, and service convenience. There has been almost no qualitative analysis of previous studies. For example, environmental factors are rarely considered, including effects of air quality and lightning strikes. In order to promote car users' love for environment and awareness of protection, it is possible to consider building charging station in a place where air quality is relatively poor. Most methods used today are multi-objective optimizations and few people apply deep learning method to the area. With arrival of the big data era, it is a matter of time before deep learning is applied to the planning field of electric vehicle charging stations. The charging stations currently in use is increasing and the data is getting better and better which providing a good environment for deep learning in this field.

\section{BP Neural Network}

The BP (error Back Propagation, BP) neural network consists of an input layer, one or more hidden layers, and an output layer. Each layer contains several neural unit nodes. One node is a learning unit. The output value is set by input values, weights, thresholds and activation functions. A simple mathematical model of a neural node:

$$
\begin{gathered}
Z_{j}=\sum_{i=1}^{n} w_{j i} X_{i}-\theta_{j} \\
y_{j}=f\left(Z_{j}\right)
\end{gathered}
$$

where, $i$ : Number of nodes in the input layer;

$j$ : Number of nodes in the output layer;

$X_{i}(i=1,2, \cdots, n)$ : Enter information;

$w_{j i}:$ Weight of two layers of neuron nodes;

$\theta_{j}:$ Threshold;

$Z_{j}$ : Total input;

$f\left(Z_{j}\right)$ : Activation function (usually with Sigmoid function); 
$y_{j}$ : Output information.

The BP algorithm is divided into two parts that is forward propagation and error back propagation shown in Figure 2.

If actual output does not match expected output, the process of error back propagation begins shown in Figure 3.

The training process of BP neural network consists of two processes: forward propagation of information and back propagation of error. Firstly, the weights between the nodes of each layer of the neural network are randomly initialized, and the input layer receives the information provided by the training samples. According to the weight of the network and the transfer function between the layers, the information is processed by forward propagation, and finally the calculation is performed. Obtaining the output value and completing a forward propagation process; when the actual output of the neural network does not match the expected output, it enters the back propagation phase of the error, and reverses the error of each layer from the output layer, using an optimization algorithm. Adjust the weight between the nodes of each layer until the output error of the network reaches the preset requirement or reaches the preset number of learning. The forward and backward propagation is the process of constant adjustment of weights and the training process of BP neural network, as shown in Figure 4.

\section{Evaluation System for Electric Vehicle Charging Station Planning}

There are many factors affecting planning of electric vehicle charging stations. In the paper, five standards such as natural environment standards, social standards, traffic standards, grid standards and economic standards, are subdivided into 15 sub-criteria, and their evaluation indicators are standardized. According to value of each sub-standard, corresponding evaluation value is given, and the higher the evaluation value is, the more favorable to the planning of the charging station. Among influence factors of charging station location, there are both qualitative and quantitative indicators and the units and standards are also variant between different influence factors. If data range input is quite different, it may lead to long training time of the neural network, slow convergence rate and large difference in the proportion of different influence factors. Therefore, the standardization of data is contributed to facilitate the training and modeling of the neural network.

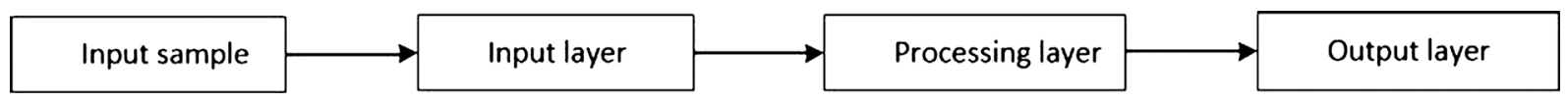

Figure 2. Forward propagation.

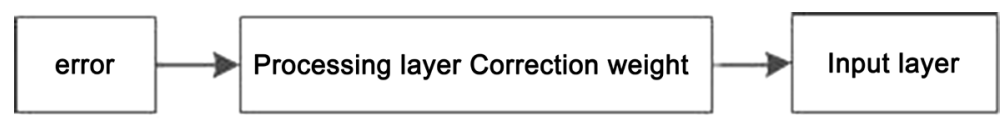

Figure 3. Error back. 


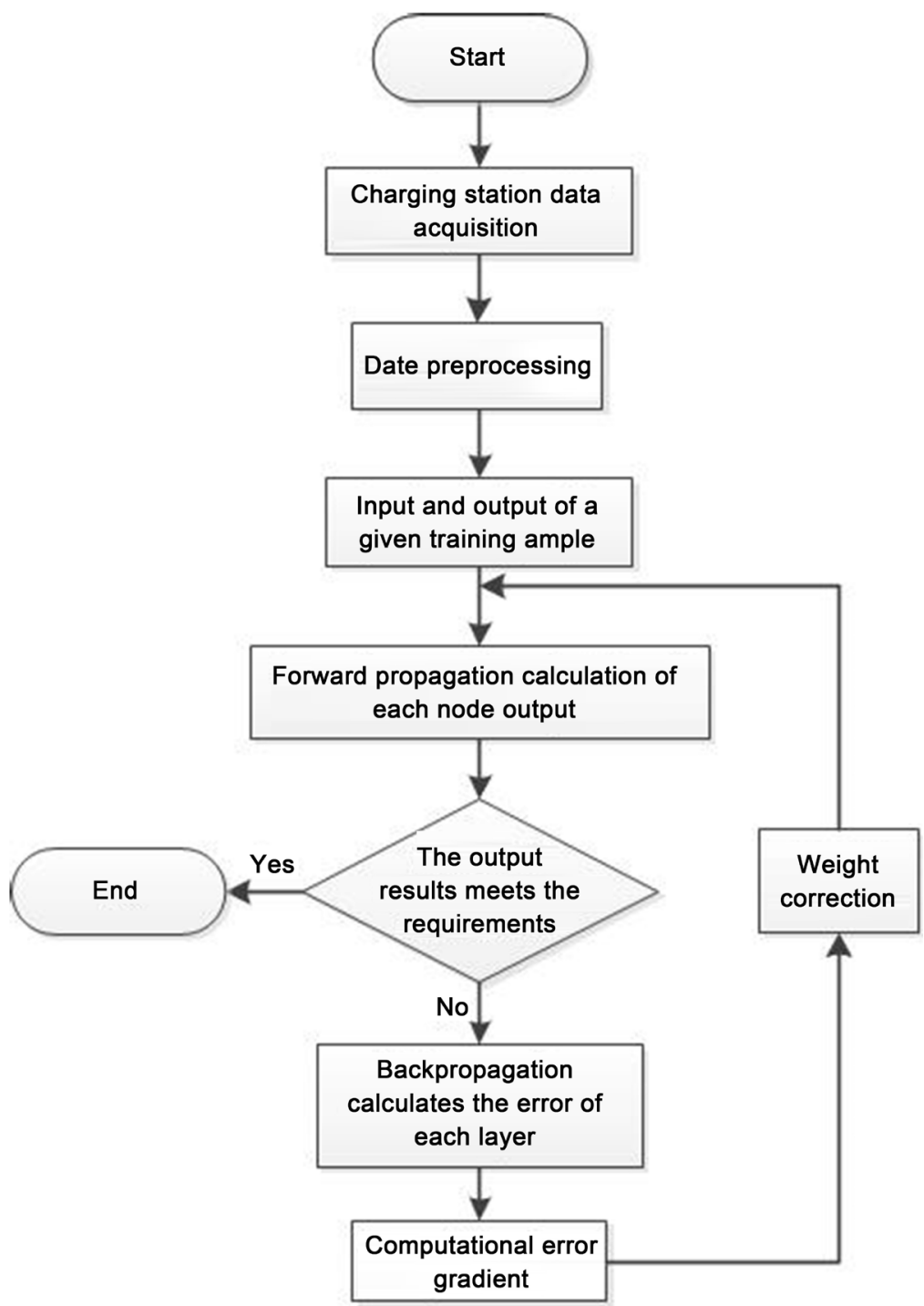

Figure 4. Modeling process of charging station planning based on BP neural network.

\subsection{Natural Environment Standard}

For the previous research issues in charging station planning, the impact of the natural environment is rarely considered. The natural environment also has a certain influence in the planning of charging stations, such as Thunderstorm Days and Air Quality Index (AQI).

As shown in Table 1, thunderstorm Day refers to numbers of days of lightning discharge in a region indicating the frequency of lightning activity in different regions. The charging station must be built in a safe place. If selected address is easily get lightning damage, it will not only threaten the safety of people, but also increase the cost of adding lightning protection function in the charging station.

As shown in Table 2 and Table 3, the Air Quality Index (AQI) is divided into six levels. As the index increases, the level increases and the pollution level increases. In order to protect the environment and raise public awareness of environmental 
protection, fuel vehicles are designed to replace fuel vehicles. Charging stations should be built in places with high pollution levels without affecting people health, which can raise public awareness of environmental protection and encourage citizens to use electric vehicles. Therefore, the evaluation values of moderate pollution and mild pollution are high and serious or severe pollution has affected people health so it is not conducive to built the charging station and then come with low evaluation value.

\subsection{Social Standard}

The social function properties and development of the area where the charging station is located have an important impact on the charging station. As shown in Table 4, it specifically includes three aspects.

Table 1. Thunderstorm day evaluation form.

\begin{tabular}{cc}
\hline Thunderstorm day & Value \\
\hline $0-20$ & 5 \\
$21-30$ & 4 \\
$31-40$ & 3 \\
$41-50$ & 2 \\
Above 50 & 1 \\
\hline
\end{tabular}

Table 2. Air quality index evaluation form.

\begin{tabular}{lcc}
\hline \multicolumn{1}{c}{ Grade } & AQI & Value \\
\hline Level 1: Excellent & $0-50$ & 3 \\
Level 2: Good & $51-100$ & 5 \\
Level 3: Mild pollution & $101-150$ & 6 \\
Level 4: Moderate pollution & $151-200$ & 2 \\
Level 5: Heavy pollution & $201-300$ & 1 \\
Level 6: Severe pollution & Above 300 & \\
\hline
\end{tabular}

Table 3. Natural environment standard evaluation form.

\begin{tabular}{ccc}
\hline Variable name & Impact factor & Value \\
\hline N1 & Thunderstorm day & $(5,4,3,2,1)$ \\
N2 & Air quality index(AQI) & $(6,5,4,3,2,1)$ \\
\hline
\end{tabular}

Table 4. Social standard evaluation form.

\begin{tabular}{clc}
\hline Variable name & \multicolumn{1}{c}{ Impact factor } & \multicolumn{1}{c}{ Value } \\
\hline S1 & $\begin{array}{l}\text { Regional functions (residential area, office area, } \\
\text { leisure area, commercial area) }\end{array}$ & $(4,3,2,1)$ \\
S2 & Regional development level (from development to maturity) & $(1,2,3)$ \\
S3 & Parking lot (yes/no) & $(2 / 1)$ \\
\hline
\end{tabular}


It takes a certain amount of time for the electric vehicle to charge, so the function of the area where the charging station is located directly affects the use of the charging station. The charging station near the residential area is the most convenient to use, charging at home time to avoid affecting the use of cars and wasting time. The charging station in the office area is also convenient for the public.

The degree of development of the region also has an impact on the charging station. The area is divided into three levels from developing to maturity, and the evaluation values are: 1,2 , and 3 .

Many charging stations are built in the parking lot, where the electric vehicle can be charged and parked as well, which not only saves land resources, but also improves the convenience of charging electric vehicles.

\subsection{Traffic Standard}

Traffic has the most important impact on the planning of the charging station. According to the data collected by the existing charging station, the number of lanes, the number of traffic lights, and the typical traffic volume are reasonably divided into sections, and the corresponding intervals are set. The specific standards are shown in Table 5.

As for the number of lanes, according to the number of lanes in the $5 \mathrm{kM}$ range of the station, the more lanes there are, the more convenient the traffic is, and the higher the evaluation value is.

The number of traffic lights and traffic lights can improve the safety and stability of traffic, but as the number of traffic lights increases, it will affect the smoothness of traffic and lower the evaluation value.

The typical traffic flow and traffic flow intuitively reflect the ability of the charging station to intercept the vehicle. As the traffic volume increases, the evaluation value increases.

\subsection{Grid Standard}

The construction of the charging station is inseparable from the grid environment and affects each other. The capacity of the charging station will affect the stability of the power grid, the stability of the power grid and the quality of service of the charging station [7]. As shown in Table 6, it is divided into 4 aspects.

The greater the loads of the recent important points are, the more favorable it is for the construction of the charging station, and the expansion of the charging station is allowed, so that the future development of the charging station will be

Table 5. Traffic standard evaluation form.

\begin{tabular}{ccc}
\hline Variable name & Impact factor & Value \\
\hline T1 & Number of lanes & $(5,4,3,2,1)$ \\
T2 & Number of traffic lights & $(5,4,3,2,1)$ \\
T3 & Typical traffic flow & $(5,4,3,2,1)$ \\
\hline
\end{tabular}


Table 6. Grid standard evaluation form.

\begin{tabular}{ccc}
\hline Variable name & Impact factor & Value \\
\hline G1 & Recent important load point level & $(4,3,2,1)$ \\
G2 & Important load point distance & $(3,2,1)$ \\
G3 & Channel resource availability margin & $(3,2,1)$ \\
G4 & The impact of charging stations on grid stability & $(2,1)$ \\
\hline
\end{tabular}

Table 7. Economic standard evaluation form.

\begin{tabular}{ccc}
\hline Variable name & Impact factor & Value \\
\hline E1 & Recent charging station distance & $(3,2,1)$ \\
E2 & Land cost & $(3,2,1)$ \\
E3 & Long-term planning (conformity/non-conformity) & $(2 / 1)$ \\
\hline
\end{tabular}

more flexible and the evaluation value will be higher.

The closer the distance to the important load point is, the lower the construction cost of the charging station is, and the higher the evaluation value is.

The available margin of channel resources is related to the future planning of the nearby power grid. The higher the available margin of the channel resources are, the higher the evaluation value is.

The degree of influence of the charging station on the grid is divided into two levels. If the charging station has a relatively large impact on the power grid, it is not conducive to the construction and operation of the charging station.

\subsection{Economic Standard}

As shown in Table 7, when the charging station is close to other charging stations, the traffic flow of the charged electric vehicles will be dispersed, and the more serious the consequences of mutual influence, the lower the evaluation value is.

High land cost will increase the construction cost of the charging station and reduce its economic efficiency. The low land cost is more conducive to the construction and operation of the charging station.

If the location cannot meet the urban planning, it may be dismantled in the near future and cause unnecessary waste and reduce economic efficiency. Therefore, it is in line with the long-term planning of the city, which is conducive to the development of the charging station, and then come with high evaluation value.

\section{Electric Vehicle Charging Station Planning Model}

\subsection{Electric Vehicle Charging Station Planning Model Construction}

Based on BP neural network, the model mainly includes the location model and capacity prediction model of the charging station. The algorithm process of the BP neural network is shown in Table 8 [8]. 
Table 8. Algorithm of BP neural network.

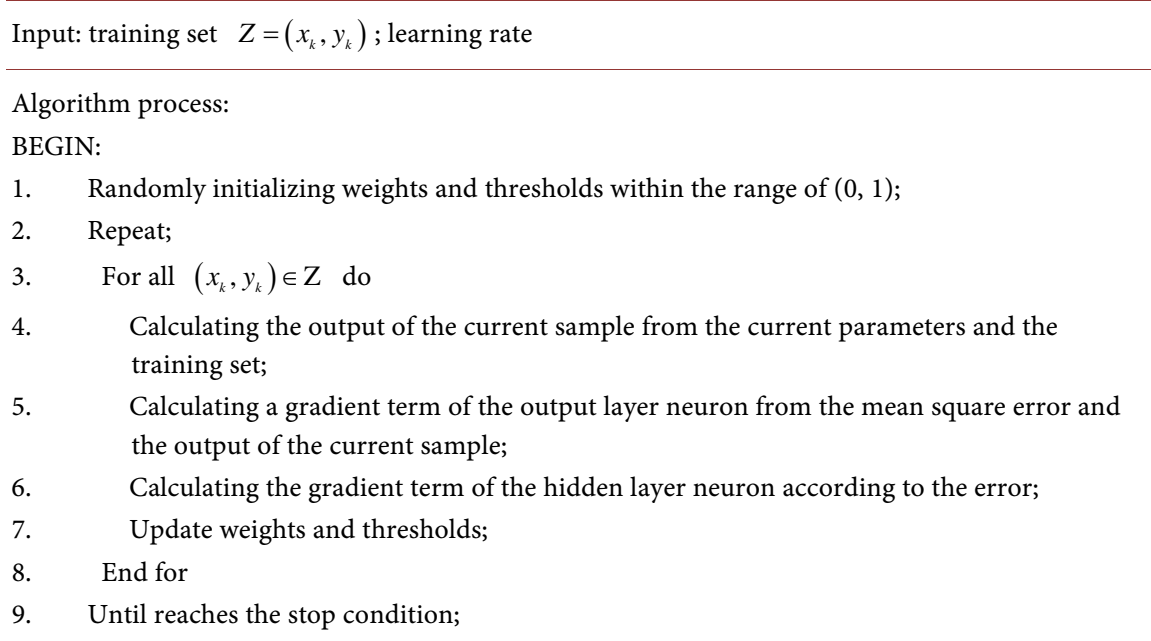

Output: charging station planning model, weighted and threshold-determined multilayer feedforward neural network

Location model: The influence factors are collected for the operation charging station and make data standardized, and then put processed data as input. The charging station is evaluated according to the actual operation of the charging station and the evaluation of the professional, and put evaluation value as an output to constitute a training set and construct a stable location neural network model.

Capacity prediction model: The processed influence factor is taken as input, actual operation capacity of charging station as output to get a training set constructing a stable capacity prediction neural network model. The data collected by the candidate station is processed and input into the location model and the capacity prediction model, and the evaluation value of the charging station candidate station location and the predicted capacity are output.

\subsection{Experiments and Applications}

In the paper, the data collected from 50 charging stations putting into operation are used as training samples. According to government' urban planning and field survey, five charging station candidate stations are selected, and the data of these five candidate stations are input into the model for prediction. According to relevant theoretical experience and many experiments, the target error is set to 0.00065 , the learning rate is 0.05 , and the maximum number of training is 50,000. After the training set is trained, the mean square error of the network reaches the optimal level after 524 iterations, and a stable charging station planning neural network model is obtained. As shown in Figure 5, the mean square error of the model achieves the desired performance. As shown in Figure 6, the regression state reached 0.99839 .

As shown in Figure 7, the red " $O$ " represents the network output evaluation value, and the blue "+" represents the actual input evaluation value. It can be 


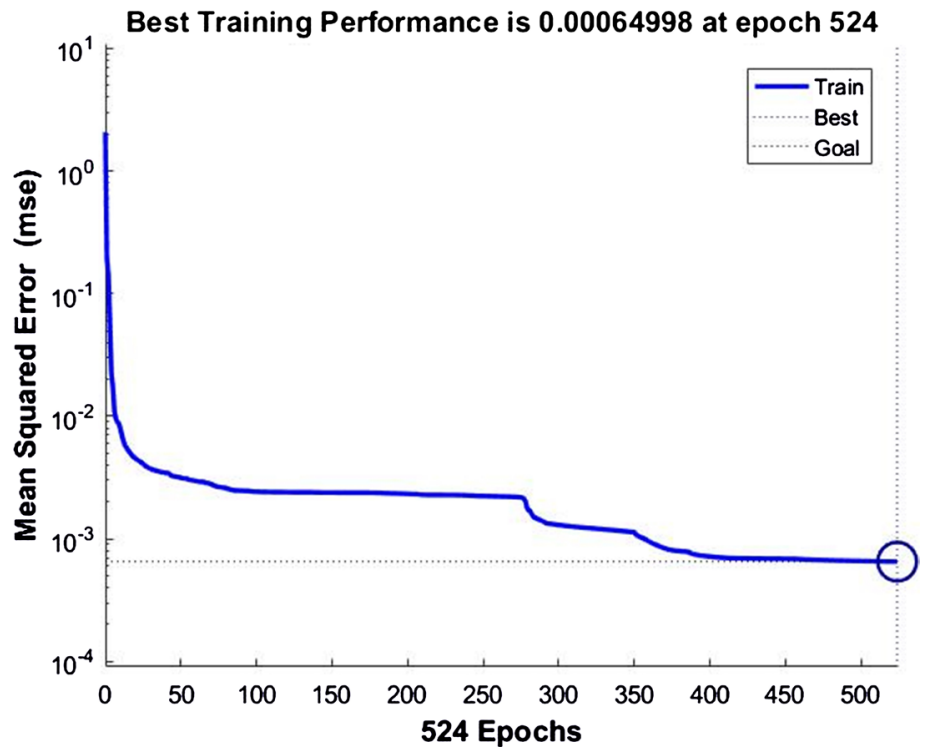

Figure 5. Training performance.

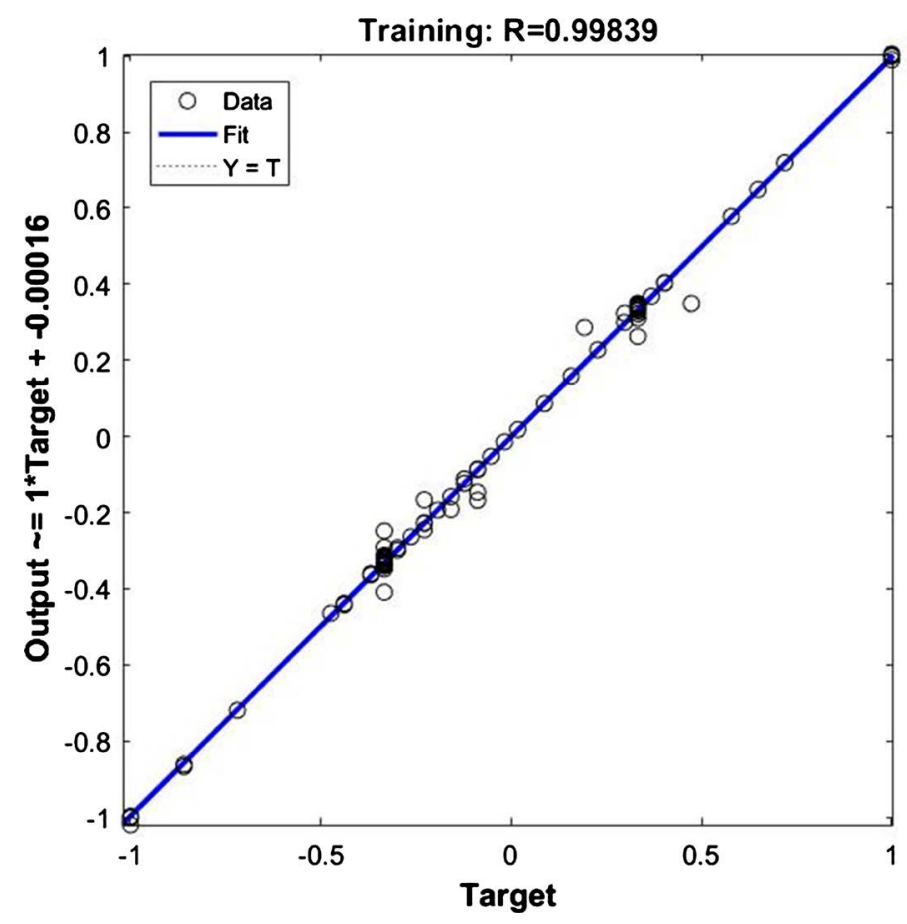

Figure 6. Regression state.

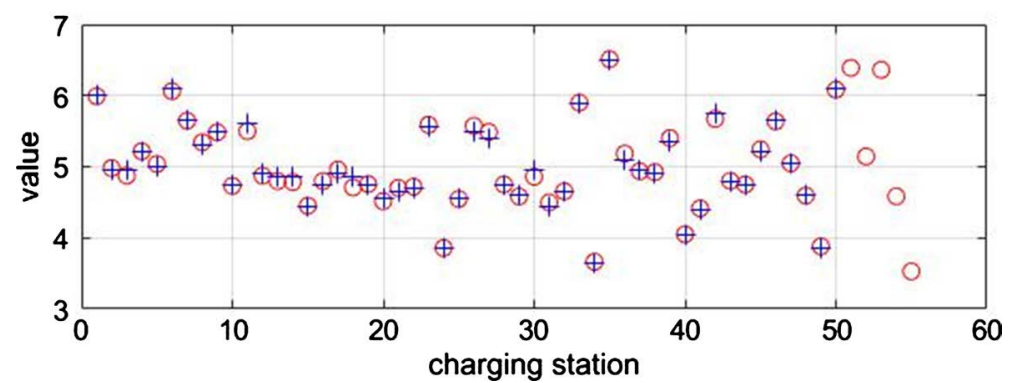

Figure 7. Training data and prediction data for charging station location evaluation values. 
seen that the network output evaluation value and the actual input evaluation value are basically the same, achieving a good learning effect. The five red "O"s at the back represent the predicted charging station evaluation values.

As shown in Figure 8, the red "O" represents the network output capacity, and the blue "+" represents the actual input capacity. It can tell that the network output capacity is same with the actual input capacity basically and achieving a good learning effect. The five red "O"s on the back represent the predicted charging station capacity.

The results of the five charging station candidate stations are shown in Table 9. According to the output results of the electric vehicle charging station planning model based on BP neural network, among the five candidate charging stations, the evaluation values $\mathrm{P} 2$ and $\mathrm{P} 4$ are the higher indicating the environment around the candidate station are suitable for the construction and operation of charging station. The result of the model output can be used as an important reference for the actual location of the charging station. According to the capacity and actual situation predicted by the model, the capacity can be selected as: $0.4 \mathrm{MW}, 0.4 \mathrm{MW}$.

\section{Conclusion}

In this paper, the influence factors are divided into five standards and 15 sub-standards affecting electric vehicle charging stations such as natural environment standards, social standards, traffic standards, power grid standards and economic standards establishing an evaluation system for charging station planning. By analyzing data, the BP neural network algorithm was introduced and data was collected and standardized based on the 15 sub-standards, through

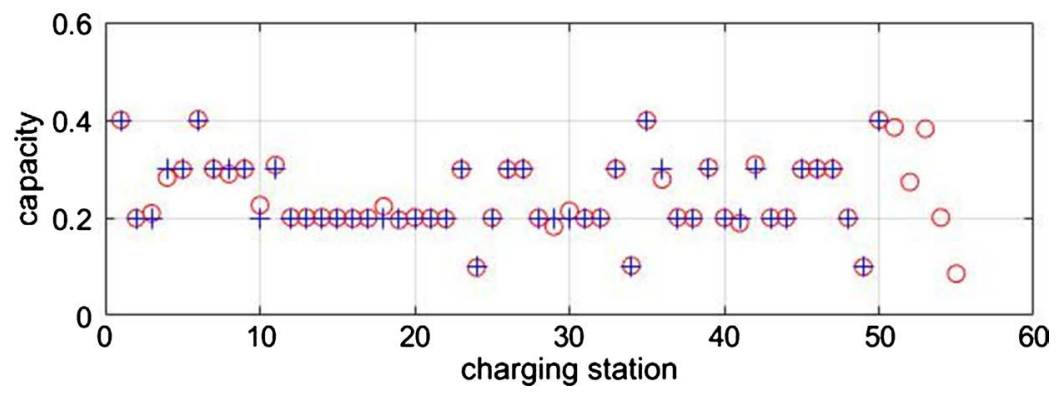

Figure 8. Training data and forecast data for charging station capacity.

Table 9. Prediction results of five candidate stations.

\begin{tabular}{ccc}
\hline Candidate station & Value & Forecast capacity (MW) \\
\hline P1 & 3.5279 & 0.0855 \\
P2 & 6.3627 & 0.3821 \\
P3 & 4.5834 & 0.2005 \\
P4 & 6.3889 & 0.3852 \\
P5 & 5.1441 & 0.2732 \\
\hline
\end{tabular}


which we can get a training set. Through BP neural network training, an electric vehicle charging station planning model is obtained based on BP neural network. Compared with the traditional multi-objective optimization method, the proposed method adopts the data of the charging station in operation, so the impact factor is more comprehensive and model is more in line with the actual operation effect of the charging station. The charging station planning problem should not only consider the quantitative indicators, but also consider the qualitative indicators. The big data is applied to the charging station's location and capacity prediction problems successfully and solve the charging station planning problem with deep learning.

\section{Conflicts of Interest}

The authors declare no conflicts of interest regarding the publication of this paper.

\section{References}

[1] Seyedmohsen, H. and Sarder, M.D. (2019) Development of a Bayesian Network Model for Optimal Site Selection of Electric Vehicle Charging Station. International Journal of Electrical Power and Energy Systems, 105, 110-122. https://doi.org/10.1016/j.ijepes.2018.08.011

[2] Zhang, J., Zhou, H. and Li, H. (2017) Multi-Objective Planning of Charging Stations Considering Vehicle Arrival Hot Map. 2017 IEEE Conference on Energy Internet and Energy System Integration, Beijing, 26-28 November 2017, 1-6. https://doi.org/10.1109/EI2.2017.8245374

[3] Yin, X. and Zhao, X. (2016) Planning of Electric Vehicle Charging Station Based on Real Time Traffic Flow. 2016 IEEE Vehicle Power and Propulsion Conference, Hangzhou, 17-20 October 2016, 1-4. https://doi.org/10.1109/VPPC.2016.7791751

[4] Wang, H., Shen, H. and Ouyang, W. (2018) Exploiting POI-Specific Geographical Influence for Point-of-Interest Recommendation. Proceedings of the 27 th International Joint Conference on Artificial Intelligence, Stockholm, 13-19 July 2018, 3877-3883. https://doi.org/10.24963/ijcai.2018/539

[5] Liu, B., Huang, X., Li, J., Qian, X. and Cheng, J. (2015) Multi-Objective Planning of Distribution Network Containing Distributed Generation and Electric Vehicle Charging Stations. Power System Technology, 39, 450-456.

[6] Yang, Z. and Gao, Z. (2018) Location Method of Electric Vehicle Charging Station Based on Data Driven. Journal of Transportation Systems Engineering and Information Technology, 18, 143-150.

[7] Cui, Q., Weng, Y. and Tan, C.-W. (2019) Electric Vehicle Charging Station Placement Method for Urban Areas. IEEE Transactions on Smart Grid, 1. https://doi.org/10.1109/TSG.2019.2907262

[8] Zhou, Z. (2016) Learning Machine. Tsinghua University Press, Beijing. 